Research Article

\title{
Ensemble Learning Prediction of Drug-Target Interactions Using GIST Descriptor Extracted from PSSM-Based Evolutionary Information
}

\author{
Xinke Zhan $\mathbb{D}^{D}$, Zhuhong You $\mathbb{D}^{\text {, }}$, Changqing Yu, Liping Li, and Jie Pan \\ School of Information Engineering, Xijing University, Xi'an 710123, China \\ Correspondence should be addressed to Zhuhong You; zhuhongyou@gmail.com
}

Received 24 June 2020; Revised 2 August 2020; Accepted 10 August 2020; Published 24 August 2020

Guest Editor: Haroon Ur Rashid

Copyright (c) 2020 Xinke Zhan et al. This is an open access article distributed under the Creative Commons Attribution License, which permits unrestricted use, distribution, and reproduction in any medium, provided the original work is properly cited.

\begin{abstract}
Identifying the drug-target interactions (DTIs) plays an essential role in new drug development. However, there still has the limited knowledge of DTIs and a significant number of unknown DTI pairs. Moreover, the traditional experimental methods have inevitable disadvantages such as high cost and time-consuming. Therefore, developing computational methods for predicting DTIs is attracting more and more attention. In this study, we report a novel computational approach for predicting DTI using GIST feature, position-specific scoring matrix (PSSM), and rotation forest (RF). Specifically, each target protein is first converted into a PSSM for retaining evolutionary information. Then, the GIST feature is extracted from PSSM and substructure fingerprint information is adopted to extract the feature of the drug. Finally, combining each protein and drug features to form a new drug-target pair, which is employed as input feature for RF classifier. In the experiment, the proposed method achieves high average accuracies of $89.25 \%, 85.93 \%, 82.36 \%$, and $73.89 \%$ on enzyme, ion channel, G protein-coupled receptors (GPCRs), and nuclear receptor, respectively. For further evaluating the prediction performance of the proposed method, we compare it with the state-of-the-art support vector machine (SVM) classifier on the same golden standard dataset. These promising results illustrate that the proposed method is more effective and stable than other methods. We expect the proposed method to be a useful tool for predicting large-scale DTIs.
\end{abstract}

\section{Introduction}

Identification of drug-target interaction (DTI) plays a vital role in researching and developing new drugs. Recently, many researchers have conducted extensive research into the DTI due to its essential role in seeking new protein to the target for drug development and promoting the emergence of new drug candidates [1,2]. However, the knowledge structure of drug-target is still incomplete, and only a small portion of target proteins of drugs have been proved as interactive. Researchers have carried out a large number of experimental methods to identify drug-target interactions, but these experimental methods have inevitable shortcomings such as time-consuming and high cost. It is known that drug development is a long process, and the whole process of introducing a new drug to market will take at least more than ten years and cost more than billions of dollars. The Food and Drug Administration (FDA) approved only a few of the drug candidates to reach the market $[3,4]$, due to many new drug candidates fail to achieve expected performance or have harmful side effects in clinical trials. Therefore, it is becoming increasingly urgent to identify drug-target interactions by developing effective new computational methods [5] which can reduce the cost and time of the experimental approach. The reliable computational method could accelerate drug discovery and potentially find some better drug candidates [6].

With the rapid development of genomics and bioinformatics, the accumulation of drug-target data is increasing. In order to store and apply data more efficiently, many related databases such as Therapeutic Target Database (TTD) [7, 8], Kyoto Encyclopedia of Genes and Genomes (KEGG) [9], SuperTarget and Matador [10], and DrugBank [11, 12] have been established. These massive data can provide abundant 
resources for researchers to study drug-target interactions and develop a novel computational approach.

Up to now, traditional calculation methods are mainly composed of ligand-based methods [13] and structurebased methods [14-16]. As for ligand-based prediction methods, it is mainly used to predict the biological activity of molecules on specific targets. However, the performance of the constructed model does not satisfy the expected requirements if the number of known active molecules of a specific target is insufficient, and the information of protein domain is unused. For the structure-based method, molecular docking is one of the most widely used methods. This method needs to study the interaction between drug molecules and target proteins through the three-dimensional (3D) structure information of known targets. At the same time, for a given drug or new chemical entity, reverse docking can be used to predict potential targets with which it interacts [17-19]. However, it is known that proteins with the 3-D structure only account for a small part of the whole proteins, which makes the method difficult to meet the requirements of experimental methods. Therefore, it is more effective to develop new protein sequence-based prediction models to predict drug-target interactions.

Until now, a number of computational methods aimed at identifying new drug-target interactions have been proposed. For example, Yamanishi et al. [20] proposed a bipartite graph learning model method, which integrates the chemical space of drugs, the gene space of proteins, and the topological information of drug target interaction network into a unified pharmacological space. Li et al. [21] proposed a novel prediction method using local binary pattern (LBP) and discriminative vector machine (DVM) for predicting DTIs. Liu et al. [22] proposed a logistic matrix factorization algorithm based on neighbour regularization, using a neighbour regularization factor to solve the problem of new drug additional. Nagamine and Sakakibara [23] developed a computational method which combined the amino acid sequence data, the chemical structure of the ligand, and mass spectrometry (MS) as input data and used the support vector machine (SVM) to build a prediction model. Meng et al. [24] reported a novel computational method, namely, PDTPS, which is aimed at predicting drug-target interactions based on protein sequences and drug chemical structures. Yu et al. [25] designed a relatively systematic method, which integrates the chemical, genomic, and pharmacological information of drugs and targets. Huang et al. [26] proposed a new computational approach for predicting DTIs. Specifically, the protein sequence is transformed into a pseudo substitution matrix representation (Pseudo-SMR) descriptor, which retained the biological evolutionary information and predicting DTIs after connecting two vector spaces of drug structure and protein sequence. In order to handle the problem of imbalance data, many computational methods [27] have been proposed which aims to solve the problem for predicting DTIs. For example, Mahmud et al. [28] presented a new computational model, namely, pdti-EssB, which constructed a predictive model with XGBoost; the model used databalancing techniques to handle the imbalance problem and adopted a novel feature eliminator for accurate prediction.
TABLE 1: The statistic of four drug-target data.

\begin{tabular}{lccc}
\hline Dataset & Drugs & Target proteins & Interactions \\
\hline Enzyme & 445 & 664 & 2926 \\
Ion channels & 210 & 204 & 1476 \\
GPCRs & 223 & 95 & 635 \\
Nuclear receptors & 54 & 26 & 90 \\
\hline
\end{tabular}

Ezzat et al. [29] proposed a novel method which is focused on addressing two imbalance problems. The first was solving the high imbalance ratio between the minority and majority classes, and the second was aimed at dealing with the within-class imbalance prevalent; the method is effective for predicting drug-target interactions.

In our work, we proposed a novel computational method, which based on drug substructure fingerprints and the information of the target protein sequence to predict drug-target interactions on a large scale. The proposed method combines GIST feature, position-specific scoring matrix (PSSM), and rotation forest (RF). The method mainly contains three steps: converting the target protein sequence into PSSM and adopting molecular substructure fingerprints as the feature of drugs are the first step, and then GIST feature vectors are extracted from PSSM. Finally, the GIST feature vectors would input to the RF classifier and obtain the result of prediction. In order to better evaluate the proposed method, a five-fold cross validation method is adopted on four golden datasets, including enzyme, ion channels, GPCRs, and nuclear receptors. Furthermore, we make a comparison between the proposed method and the state-of-the-art support vector machine (SVM) classifier on enzyme dataset, and we also compare the result of the proposed method with previous work on four datasets. The promising results show that our method is efficient and robust to predict drug-target interactions.

\section{Materials and Methods}

2.1. Golden Standard Datasets. In this study, four golden standard datasets, including enzymes, ion channels, GPCRs, and nuclear receptors were explored by using the proposed method for evaluating the prediction ability of drug-target interactions. All these datasets were freely available from BRENDA [30], DrugBank [11], KEGG BRITE [9], and SuperTarget [10] databases, and these drug-target datasets from high-reliability databases are generally considered the golden standard datasets. The number of drugs known to target enzyme, ion channels, GPCRs, and nuclear receptors is $445,210,233$, and 54, respectively. The number of proteins targeted by the drugs is known to be 664, 204, 95, and 26, respectively. In these total datasets, 5127 drug-target pairs were known to interact with each other. These data are distributed over enzyme, ion channels, GPCRs, and nuclear receptors, respectively, and the number of them are 2926, 1476,635 , and 90, respectively. Table. 1 summarizes the statistics of the number of four drug-target datasets.

Generally speaking, we usually consider a drug-target interaction network as a bipartite graph in which nodes 
describe drug molecules or target proteins and edges represent the relationship between the nodes. It was very sparse of the initial bipartite graph of drug-target interactions for which only a small fraction of the real drug-target interactions edges have been validated by experimental method or other ways. Take the enzyme dataset as an example, and there are 295,480 $(445 \times 664)$ connections in the corresponding bipartite graph in total. However, only 2926 initial edges which account for only $1.00 \%$ of the total connections were known drug-target interactions. These known interaction pairs were treated as positive samples (2926) which were obviously less than the possible number of negative samples $(295,480-2926=292,554)$. There exists a bias problem caused by the unbalance samples. In order to deal with this problem, the number of negative samples was selected randomly as much as the positive samples. It would know that the real interaction negative samples we have chosen is quite small or even can be ignored when study a large-scale of DTI. As a result, the negative samples of enzyme, ion channels, GPCRs, and nuclear receptors datasets were 2926, 1476, 635, and 90 , respectively.

2.2. Molecular Substructure Fingerprint of Drug. Many kinds of research have shown that drug compounds could be represented as different types of descriptor such as constitutional, topological, quantum chemical properties and geometrical. Here, molecular fingerprints are being employed to represent drug compounds [31] which structure information can be effectively described by molecular fingerprints. Moreover, the calculation of molecular fingerprint only needs twodimensional structure, which not only reduces the workload of molecular descriptor calculation and screening but also avoids the error transfer and accumulation in the process of molecular descriptor calculation. According to detecting the existence of specific structural segments in the molecular structure of drug compounds, these fragments then are encoded on the corresponding bits of the corresponding binary string through a substructure pattern of a predefined dictionary, and the molecular structure is transformed into an orderly digital fingerprint sequence. Specifically, the specific structural segment exists in the given drug molecules; the corresponding bit of the vector is set to 1 , or 0 would be set. In this work, the chemical structure fingerprints set can be downloaded from the PubChem website (https://pubchem.ncbi.nlm.nih.gov/). There are 881 substructure information recorded in drug fingerprint. As a result, the molecular feature of the drug is 881 binary vectors.

2.3. Position-Specific Scoring Matrix. There exist many effective methods to transform protein sequences into multidimensional feature vectors such as using physicochemical of amino acids [32, 33] and using statistical distributions of amino acids $[34,35]$. In this work, we adopt positionspecific scoring matrix (PSSM) [36], which was adopted for exploring distantly related protein. PSSM is also widely adopted in previous work such as protein secondary structural prediction, protein binding site prediction, and protein subcellular localization. Through using a Position-Specific Iterated Basic Local Alignment Search Tool (PSI-BLAST)
[37] to search and compare the homologous sequence of each target protein sequence, the homologous information of alignment sequence can be expressed as PSSM which construct is an $M \times 20$ score matrix, where $M$ rows are the total number of amino acid sequence and 20 columns represent the number of 20 amino acid. Here, a PSSM can be obtained according to the following formula:

$$
\operatorname{PSSM}_{P}=\left(M_{1}, M_{2}, \cdots, M_{i}, \cdots, M_{20}\right),
$$

where $M_{i}=\left(M_{1, i}, M_{2, i}, \cdots, M_{n, i}, \cdots, M_{X, i}\right)^{T},(i=1,2, \cdots, 20)$. $X$ denotes the length of an amino acid sequence, and $M_{n, i}$ is the mutation score which represents the probability of amino acid $i$ residue to change into amino acid $j$ in the process of biological evolution. In this experiment, the PSI-BLAST tool was employed to transform each protein sequence into a PSSM. For obtaining highly homologous sequence, the parameter of critical value of $e$ value is set to 0.001 and maximum number of iterations is 3 ; other parameters were set to default values. For details on the use of PSI-BLAST can be obtained at https://BLAST.ncbi.nlm.nih.gov/BLAST.cgi.

2.4. GIST Feature Descriptor. The GIST feature which is a biological heuristic feature was first proposed by Oliva et al. [38]. The GIST feature can extract global feature information which plays an important role in scene image classification [39], and it has been proven to be feasible in objective recognition [40, 41] as well. In GIST algorithm, the processes mainly contain the following two steps to extract GIST feature:

(1) Creating Gabor Filters. In image processing, the Gabor filter can extract a feature from the gray-level images directly. In spatial domain, the two-dimensional Gabor filter is a Gaussian kernel function modulated by complex sinusoidal plane wave. The definition is as follows:

$G(x, y)=\exp \left(-\frac{x^{\prime 2}+\gamma^{2} y^{\prime 2}}{2 \sigma^{2}}\right) \exp \left(i\left(2 \pi \frac{x^{\prime}}{\lambda}+\psi\right)\right)$

$$
\begin{aligned}
& \text { Real part : } G(x, y) \\
& \quad=\exp \left(-\frac{x^{\prime 2}+\gamma^{2} y^{\prime 2}}{2 \sigma^{2}}\right) \cos \left(2 \pi \frac{x^{\prime}}{\lambda}+\psi\right),
\end{aligned}
$$

$$
\begin{aligned}
& \text { Imaginary part : } G(x, y) \\
& \qquad=\exp \left(-\frac{x^{\prime 2}+\gamma^{2} y^{\prime 2}}{2 \sigma^{2}}\right) \sin \left(2 \pi \frac{x^{\prime}}{\lambda}+\psi\right)
\end{aligned}
$$

where $x^{\prime}=x \cos \theta+y \sin \theta$ and $y^{\prime}=-x \sin \theta+y \cos \theta, \sigma$ is the standard deviation of Gauss function, $\psi$ represents the phase shift, $\lambda$ is the wavelength which value is specified in pixels, and $\theta$ is the direction of the parallel strips. Here, 32 Gabor filters were generated by adopting four scales and eight orientations. 
(2) Obtaining GIST Feature. Given a training sample $T$ which size is $a \times b$. A sample $X$ is divided into $n_{a} \times n_{b}$ blocks; each block is of equal size which construction can be defined as $a^{*} \times b^{*}$, where $a^{*}=a / n_{a}$ and $b^{*}=b / n_{b}$. Each block is processed by employing a set of filter banks that contain 32 Gabor filters, and the processed features are combined to form the block feature, which is called a block GIST feature. The feature vectors of a block processed by each filter are averaged, and these feature vectors are combined into a row vector, which are connected in series to obtain the final GIST feature, we can be defined as follows:

$$
G_{\text {vector }}=\left[\overline{G_{1}}, \overline{G_{2}}, \cdots, \overline{G_{i}}, \cdots, \overline{G_{32}}\right] \text {, }
$$

where $\overline{G_{i}}=\left(1 /\left(a^{*} \times b^{*}\right)\right) \sum G_{i}(x, y)$ and the dimension of $G_{\text {vector }}$ is $32 \times a^{*} \times b^{*}$. These feature vectors summarize the gradient information such as scale and orientation for different parts of a given sample [42, 43].

In this paper, each PSSM is divided into 16 regions $4 \times 4$ grids, using 32 Gabor filter banks with four scales and eight directions to extract the GIST feature of PSSM of each protein sequence. Finally, a $512(16 \times 32)$ dimensional GIST feature of each PSSM is obtained by connecting the $16(4 \times 4)$ average values of all 32 features maps.

2.5. Rotation Forest (RF) Classifier. The rotation forest algorithm was proposed by Rodriguez et al. [44]. This algorithm was based on the idea of feature transformation and focuses on improving the difference and accuracy of the base classifier. The sample set is randomly divided into $K$ subsets before each subset is drawn, and the principal component analysis (PCA) method is used to perform feature transformation on the divided subsets which is aimed at maintaining the effective of data. It not only makes each subset different but also plays a certain role in data preprocessing. Hence, the rotation forest can further improve the diversity in the ensemble and enhance the accuracy of the base classifier.

Suppose that $w=\left[w_{1}, w_{2}, \cdots, w_{n}\right]$ contains $n$ features of a sample. Let $W$ be the training sample set which size is $N \times n$, where $N$ denotes the number of samples. Let $H$ be the feature set, and the corresponding label be the $Y=$ $\left[y_{1}, y_{2}, \cdots, y_{n}\right]^{T}$. The feature set is randomly divided into $K$ equal subsets. Suppose the number of decision trees is $L$, which can be denoted as $T_{1}, T_{2}, \cdots T_{L}$, respectively. The construction steps of the rotation forest classifier are as follows:

(1) Select the suitable parameter $K$, the feature set $H$ is randomly divided into $K$ subsets, each subset contains $(n / K)$ features

(2) Let $H_{i j}$ denote the $j$ th subfeature set of the training set, which is used to train the $i$ th classifier $T_{i}$. For each subset, a new training set $W_{i j}^{\prime}$ is generated after a bootstrap resampling with 75 percent of training set $W$
(3) Apply principal component analysis (PCA) on $W_{i j}^{\prime}$ to produce the coefficients in matrix $P_{i j}$, which is a matrix of $M \times 1$. $P_{i j}$ can be represented as $b_{i j}^{(1)}, \cdots$, $b_{i j}^{\left(\mathrm{M}_{\mathrm{j}}\right)}$

(4) The coefficients obtained in the matrix $P_{i j}$ are constructed a sparse rotation matrix $S_{i}$, which is shown as follows:

$S_{i}=\left[\begin{array}{cccc}b_{i 1}^{(1)}, \cdots, b_{i 1}^{\left(M_{1}\right)} & 0 & \cdots & 0 \\ 0 & b_{i 2}^{(1)}, \cdots, b_{i 2}^{\left(M_{2}\right)} & \cdots & 0 \\ \vdots & \vdots & \ddots & \vdots \\ 0 & 0 & \cdots & b_{i K}^{(1)}, \cdots, b_{i K}^{\left(M_{K}\right)}\end{array}\right]$.

During the prediction period, given a sample $w$, let $d_{i j}$ $\left(\mathrm{wS}_{i}^{b}\right)$ be the probability which predicted whether $w$ belongs to $y_{i}$ by the classifier $T_{i}$. Then, calculate the confidence of the class by means of the average combination, and the formula is as follows:

$$
\lambda_{j}(w)=\frac{1}{L} \sum_{i=1}^{L} d_{i j}\left(\mathrm{wS}_{i}^{b}\right) .
$$

The test sample $w$ will be assigned the category with the greatest possible.

\section{Results and Discussion}

3.1. Evaluation Criteria. Evaluation criteria play an effective role in evaluating the computational method. In this paper, we adopted the following criteria which include accuracy (Acc.), precision (Prec.), sensitivity (Sen.), and Matthews correlation coefficient (MCC). The definition is as follows:

$$
\begin{aligned}
\text { Acc. } & =\frac{\mathrm{TN}+\mathrm{TP}}{\mathrm{TN}+\mathrm{TP}+\mathrm{FN}+\mathrm{FP}}, \\
\text { Prec. } & =\frac{\mathrm{TP}}{\mathrm{TP}+\mathrm{FP}}, \\
\text { Sen. } & =\frac{\mathrm{TP}}{\mathrm{TP}+\mathrm{FN}}, \\
\mathrm{MCC} & =\frac{\mathrm{TN} \times \mathrm{TP}-\mathrm{FN} \times \mathrm{FP}}{\sqrt{(\mathrm{TN}+\mathrm{FN}) \times(\mathrm{TP}+\mathrm{FP}) \times(\mathrm{TN}+\mathrm{FP}) \times(\mathrm{FN}+\mathrm{TP})}},
\end{aligned}
$$

where true negative (TN) represents the number of drugtarget pairs that are classified as noninteracting pairs correctly, true positive (TP) denotes the count of drug-target pairs that are classified as interacting pairs correctly, false negative (FN) represents the number of samples that are classified as noninteracting pairs incorrectly, and false positive (FP) is the count of samples that are classified as interacting 
TABLE 2: 5-fold cross-validation results were generated through the proposed method on the enzyme dataset.

\begin{tabular}{|c|c|c|c|c|c|c|}
\hline Testing set & Accuracy (\%) & Precision (\%) & Sensitivity (\%) & MCC (\%) & AUC & AUPR \\
\hline 1 & 88.89 & 89.70 & 87.86 & 80.24 & 0.9570 & 0.8579 \\
\hline 2 & 89.66 & 91.07 & 88.14 & 81.45 & 0.9450 & 0.8920 \\
\hline 3 & 88.63 & 88.87 & 88.10 & 79.85 & 0.9444 & 0.8803 \\
\hline 4 & 89.91 & 91.97 & 87.20 & 81.83 & 0.9538 & 0.8728 \\
\hline 5 & 89.15 & 91.88 & 86.13 & 80.62 & 0.9391 & 0.8788 \\
\hline Average & $89.25 \pm 0.53$ & $90.70 \pm 1.37$ & $87.48 \pm 0.85$ & $80.80 \pm 0.83$ & $0.9479 \pm 0.0074$ & $0.8763 \pm 0.0125$ \\
\hline
\end{tabular}

TABLE 3: 5-fold cross-validation results were generated through the proposed method on the ion channel dataset.

\begin{tabular}{|c|c|c|c|c|c|c|}
\hline Testing set & Accuracy (\%) & Precision (\%) & Sensitivity (\%) & MCC (\%) & AUC & AUPR \\
\hline 1 & 85.59 & 86.76 & 84.12 & 75.33 & 0.9292 & 0.8435 \\
\hline 2 & 86.95 & 87.63 & 86.15 & 77.30 & 0.9431 & 0.8686 \\
\hline 3 & 87.63 & 88.18 & 87.29 & 78.31 & 0.9323 & 0.8633 \\
\hline 4 & 85.59 & 83.16 & 87.59 & 75.31 & 0.9370 & 0.8056 \\
\hline 5 & 83.90 & 86.01 & 81.73 & 72.96 & 0.9146 & 0.8287 \\
\hline Average & $85.93 \pm 1.44$ & $86.35 \pm 1.96$ & $85.38 \pm 2.45$ & $75.84 \pm 2.07$ & $0.9312 \pm 0.0107$ & $0.8419 \pm 0.0258$ \\
\hline
\end{tabular}

TABLE 4: 5-fold cross-validation results were generated through the proposed method on the GPCR dataset.

\begin{tabular}{|c|c|c|c|c|c|c|}
\hline Testing set & Accuracy (\%) & Precision (\%) & Sensitivity (\%) & MCC (\%) & AUC & AUPR \\
\hline 1 & 83.86 & 81.89 & 85.25 & 72.91 & 0.8853 & 0.7820 \\
\hline 2 & 81.89 & 79.37 & 83.33 & 70.29 & 0.8894 & 0.7493 \\
\hline 3 & 78.74 & 84.75 & 73.53 & 66.35 & 0.8660 & 0.8154 \\
\hline 4 & 85.04 & 88.29 & 79.67 & 74.34 & 0.8998 & 0.8591 \\
\hline 5 & 82.28 & 82.48 & 84.33 & 70.71 & 0.8992 & 0.7993 \\
\hline Average & $82.36 \pm 2.39$ & $83.35 \pm 3.36$ & $81.22 \pm 4.79$ & $70.92 \pm 3.04$ & $0.8879 \pm 0.0138$ & $0.8010 \pm 0.0407$ \\
\hline
\end{tabular}

TABLE 5: 5-fold cross-validation results were generated through the proposed method on the nuclear receptor dataset.

\begin{tabular}{|c|c|c|c|c|c|c|}
\hline Testing set & Accuracy (\%) & Precision (\%) & Sensitivity (\%) & MCC (\%) & AUC & AUPR \\
\hline 1 & 75.00 & 76.92 & 62.50 & 60.78 & 0.7688 & 0.6444 \\
\hline 2 & 80.56 & 78.95 & 83.33 & 68.62 & 0.8148 & 0.7007 \\
\hline 3 & 69.44 & 81.25 & 61.90 & 56.69 & 0.8254 & 0.8484 \\
\hline 4 & 72.22 & 60.00 & 85.71 & 58.61 & 0.8442 & 0.7507 \\
\hline 5 & 72.22 & 72.00 & 85.71 & 56.06 & 0.7524 & 0.7053 \\
\hline Average & $73.89 \pm 4.21$ & $73.82 \pm 8.45$ & $75.83 \pm 12.48$ & $60.15 \pm 5.08$ & $0.8011 \pm 0.0389$ & $0.7299 \pm 0.0762$ \\
\hline
\end{tabular}

pairs incorrectly. Meanwhile, we computed the receiver operating characteristic (ROC) curve, the precision-recall (PR) curve, the area under a ROC curve (AUC), and the area under precision-recall curve (AUPR) for evaluating the performance of the proposed method visually. Due to the imbalanced dataset, it is more significantly to evaluate the proposed by employing AUPR in this study.

3.2. Performance of the Proposed Method. To better verify the performance of the proposed method in this study, we adopt the five-fold cross-validation method on different types of protein target datasets: enzyme, ion channel, GPCRs, and nuclear receptor. Specifically, the whole dataset would be separated into five parts that four datasets are used for training and one dataset is used for testing. By doing this, five training models would be generated for training datasets, and GIST feature vectors and the corresponding label would be the input data of the prediction model. Finally, the prediction score could be obtained for evaluating the interaction between drug and target protein. In addition, for the sake of fairness for all experiments in this work, the corresponding parameters $K$ and $L$ of the rotation forest-based classifier were set the same. The parameter $K$ is set to be 10 , and $L$ is set to be 12 . Here, $L$ denotes the number of decision trees and $K$ means the number of feature subsets. The prediction results of the proposed method by using the five-fold crossvalidation method of enzyme, ion channel, GPCRs, and nuclear receptor datasets are listed in Tables 2-5. 


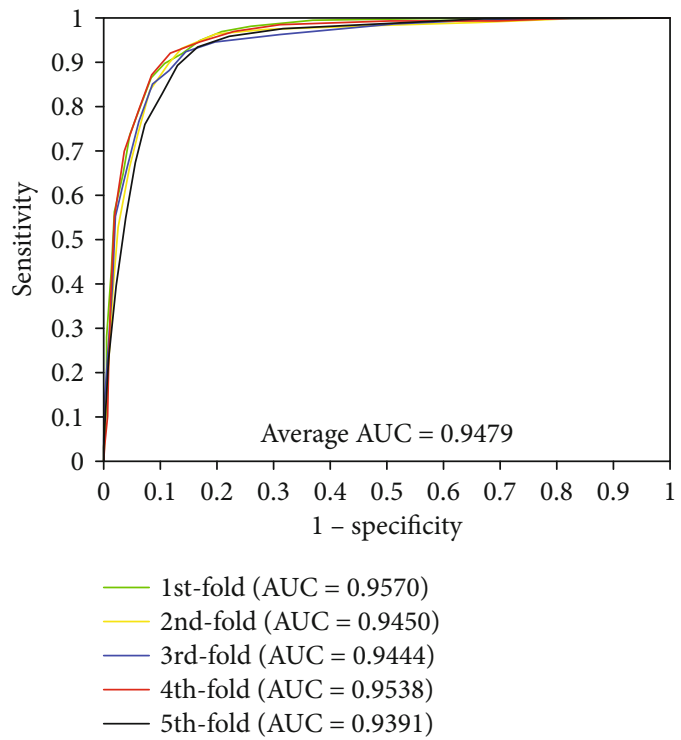

(a)

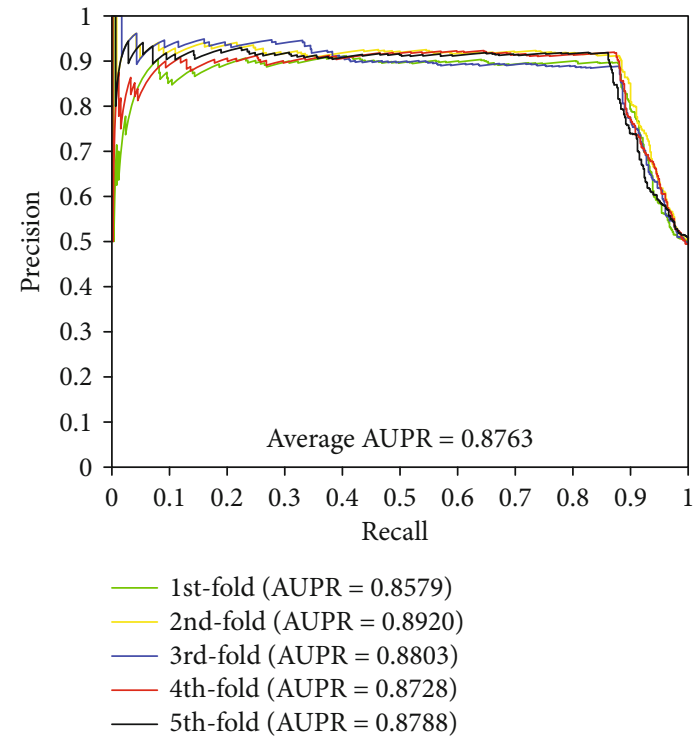

(b)

FIgURE 1: The curves obtained by the proposed method on the enzyme dataset: (a) ROC curves and (b) PR curves.

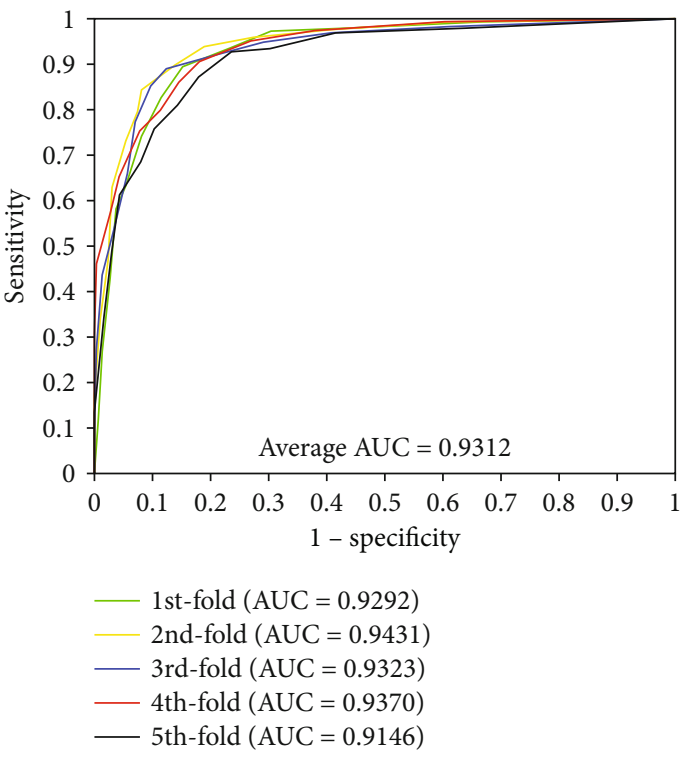

(a)

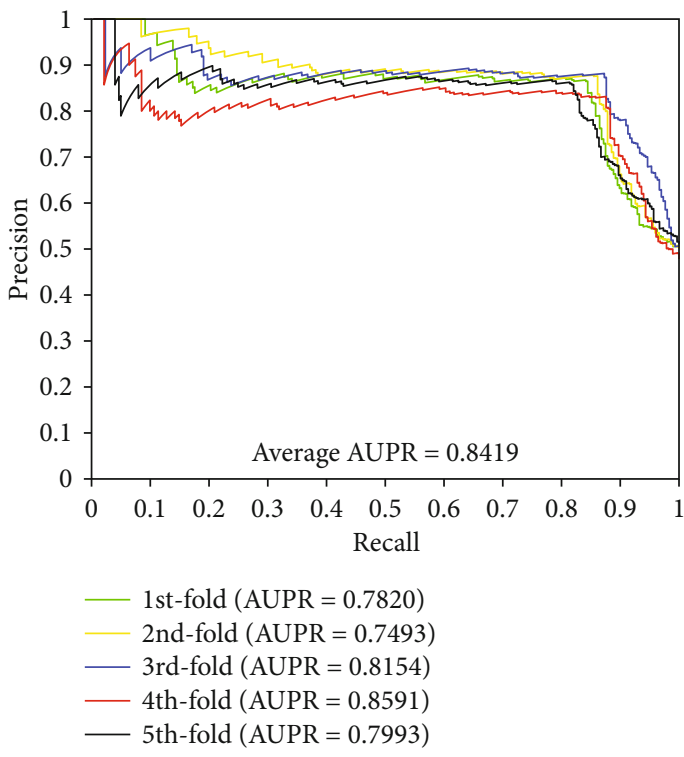

(b)

Figure 2: The curves obtained by the proposed method on the ion channel dataset: (a) ROC curves and (b) PR curves.

When our method is used to predict DTI of the enzyme dataset, the results of average accuracy, precision, sensitivity, MCC, and AUC are $89.25 \%, 90.70 \%, 87.48 \%, 80.80 \%$, and 0.9479 , respectively. The standard deviations of these predicted results are $0.53 \%, 1.37 \%, 0.85 \%, 0.83 \%$, and 0.0074 , respectively. When employing our method to predict DTI of the ion channel dataset, the results of average accuracy, precision, sensitivity, MCC, and AUC come to be $85.93 \%$, $86.35 \%, 85.38 \%, 75.84 \%$, and 0.9312 and the standard deviations are $1.44 \%, 1.96 \%, 2.45 \%, 2.07 \%$, and 0.0107 , respectively. When exploring the GPCRs dataset, the prediction result of the average accuracy, precision, sensitivity, MCC, and AUC are $82.36 \%, 83.35 \%, 81.22 \%, 70.92 \%$, and 0.8879 , respectively. The standard deviations come to be $2.39 \%$, $3.36 \%, 4.79 \%, 3.04 \%$, and 0.0138 , respectively. When predicting the interactions of the nuclear receptor dataset, we achieved the average result of accuracy, precision, sensitivity, MCC, and AUC of $73.89 \%, 73.82 \%, 75.83 \%, 60.15 \%$, and 0.8011 , respectively. It is noteworthy that the prediction result yields high standard deviations due to the samples of nuclear receptor dataset is only 90 which is smaller than the other three datasets, the standard deviations were $4.21 \%$, $8.45 \%, 12.48 \%, 5.08 \%$, and 0.0389 , respectively. Furthermore, the values of AUPR were computed on enzyme, ion channel, GPCRs, and nuclear receptor, which achieved the result of $0.8763,0.8419,0.80101$, and 0.7299, respectively. Meanwhile, 


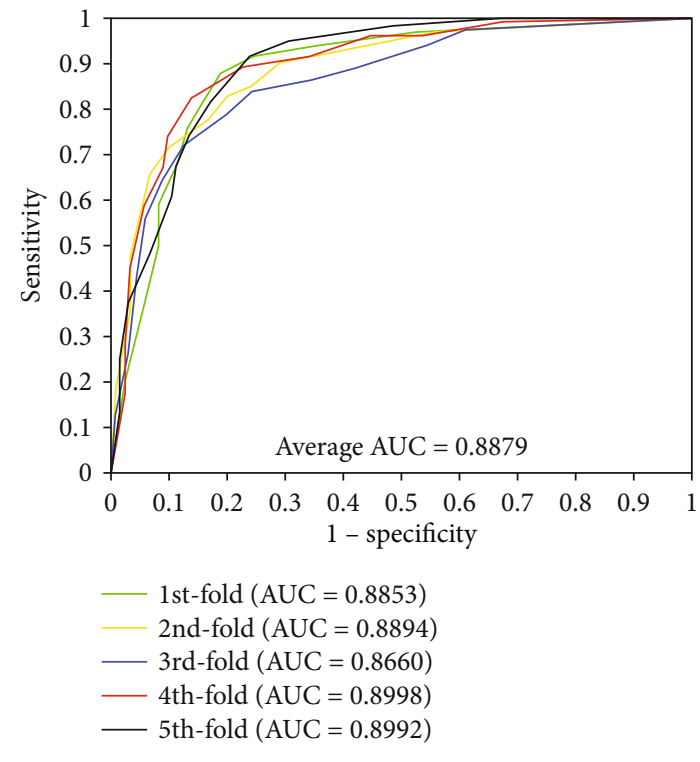

(a)

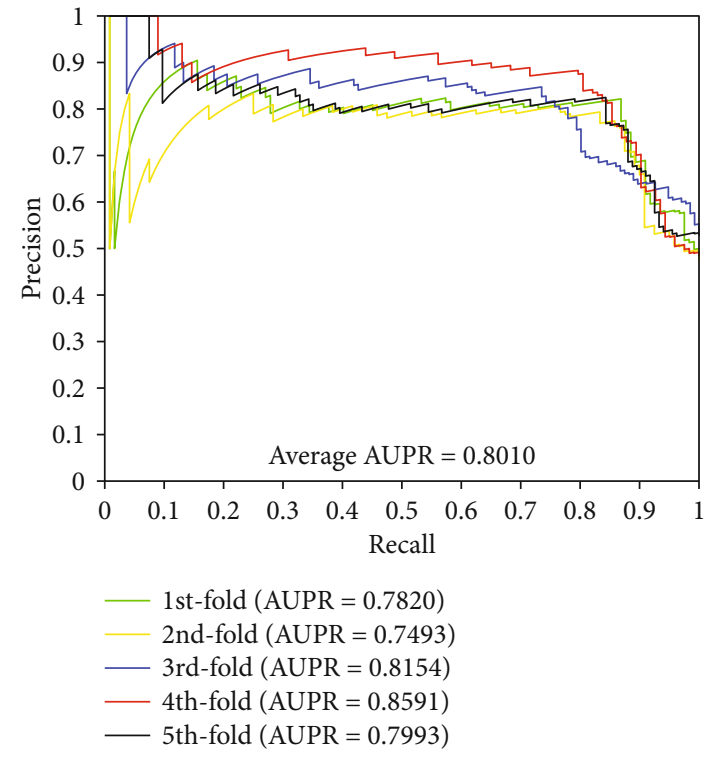

(b)

Figure 3: The curves obtained by the proposed method on the GPCR dataset: (a) ROC curves and (b) PR curves.

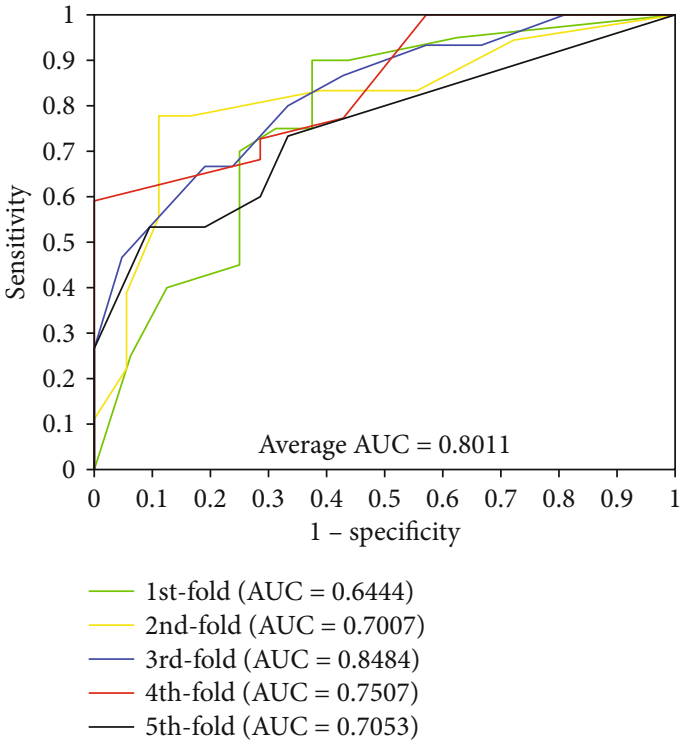

(a)

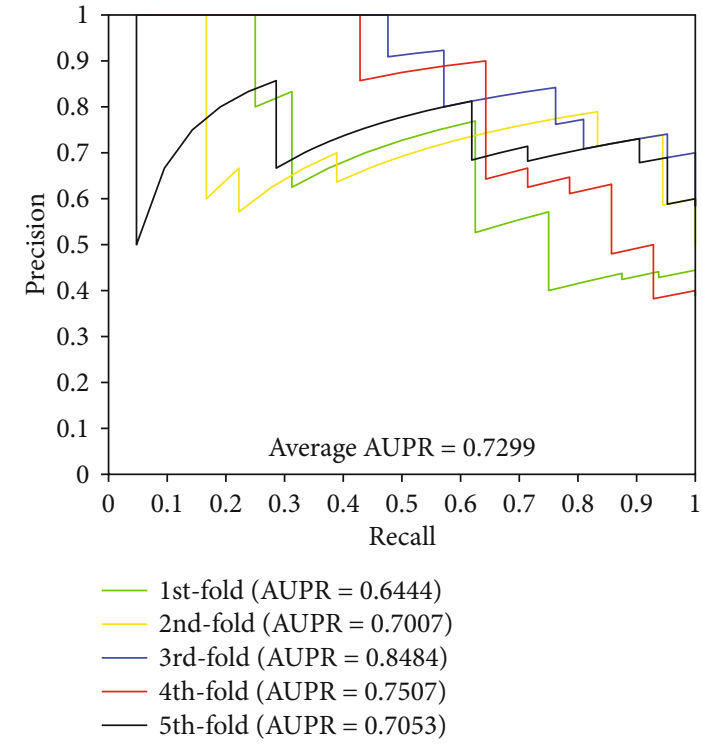

(b)

FIgURE 4: The curves obtained by the proposed method on the nuclear receptor dataset: (a) ROC curves and (b) PR curves.

the ROC curves and the PR curves of the proposed method of four datasets are shown in Figures 1-4 in order to better analyse the feasibility of the proposed method.

3.3. Comparison between RF Classifier and SVM Classifier. In order to further evaluate the prediction performance of the proposed method, we conducted the performance comparison between the RF classifier and the state-of-the-art support vector machine (SVM) classifier [45] by using the same feature descriptor vectors. We employed the five-fold crossvalidation method for better analysis at the same time. The LIBSVM tool [46] was adopted to implement classification.
We got the optimized parameters of the SVM classifier, and the parameter $c$ is set to 15 and $g$ is set to 30 . The classification result of the enzyme dataset between the RF classifier and SVM classifier is listed in Table 6. It can be seen that the result of SVM classification of average accuracy, precision, sensitivity, MCC, and AUC of $81.83 \%, 83.34 \%, 79.54 \%$, $70.23 \%$, and 0.8836 , and these standard deviation comes to be $0.64 \%, 0.78 \%, 1.16 \%, 0.83 \%$, and 0.0059 , respectively. From Table 6, we can see that the average results of the SVM classifier are lower than the performance of the proposed method. The prediction result also shows that the performance of the RF classifier is better than the performance 
TABLE 6: 5-fold cross-validation results were generated by using the proposed RF classifier and SVM classifier on the enzyme dataset.

\begin{tabular}{|c|c|c|c|c|c|}
\hline Testing set & Accuracy (\%) & Precision (\%) & Sensitivity (\%) & MCC (\%) & AUC \\
\hline \multicolumn{6}{|c|}{$\mathrm{PSSM}+\mathrm{GIST}+\mathrm{RF}$} \\
\hline 1 & 88.89 & 89.70 & 87.86 & 80.24 & 0.9570 \\
\hline 2 & 89.40 & 89.90 & 88.98 & 81.05 & 0.9450 \\
\hline 3 & 88.63 & 88.87 & 88.10 & 79.85 & 0.9444 \\
\hline 4 & 89.91 & 91.97 & 87.20 & 81.83 & 0.9538 \\
\hline 5 & 89.15 & 91.88 & 86.13 & 80.62 & 0.9391 \\
\hline Average & $89.20 \pm 0.49$ & $90.46 \pm 1.39$ & $87.65 \pm 1.07$ & $80.72 \pm 0.76$ & $0.9479 \pm 0.0073$ \\
\hline \multicolumn{6}{|c|}{ PSSM+GIST+SVM } \\
\hline 1 & 81.11 & 82.85 & 78.46 & 69.32 & 0.8794 \\
\hline 2 & 82.65 & 83.65 & 81.53 & 71.31 & 0.8895 \\
\hline 3 & 82.22 & 83.94 & 79.31 & 70.71 & 0.8901 \\
\hline 4 & 81.28 & 82.23 & 79.24 & 69.53 & 0.8820 \\
\hline 5 & 81.88 & 84.02 & 79.19 & 70.29 & 0.8770 \\
\hline Average & $81.83 \pm 0.64$ & $83.34 \pm 0.78$ & $79.54 \pm 1.16$ & $70.23 \pm 0.83$ & $0.8836 \pm 0.0059$ \\
\hline
\end{tabular}

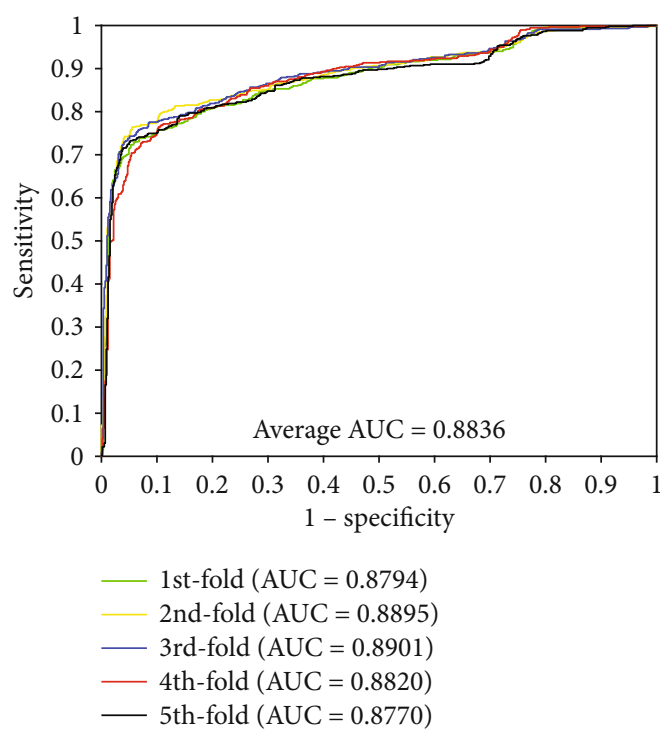

FIgURE 5: The ROC curves performed by SVM classifier on the enzyme dataset.

of the SVM classifier when employing the same feature vectors as the input data. Furthermore, the parameter optimization of the RF classifier is more convenient than the SVM classifier. Meanwhile, the ROC curves of the SVM classifier are displayed in Figure 5.

3.4. Comparison with Other Methods. Until now, numerous computational methods have been proposed for predicting the DTI. In our study, we made a performance comparison between the proposed method and the other four existing methods that include NetCBP [47], Mousavian et al.'s [48], Li et al.'s [21], and RFDTI [49]; these methods were also employed the five-fold cross-validation on enzyme, ion channel, GPCRs, and nuclear receptor dataset, respectively. The differences of them were the different feature extractions and classifiers adopted. These comparison results are listed
TABLE 7: Comparison of the AUC values between the proposed method and other four existing methods on four datasets.

\begin{tabular}{lccccc}
\hline Dataset & $\begin{array}{c}\text { Our } \\
\text { method }\end{array}$ & NetCBP & $\begin{array}{c}\text { Mousavian } \\
\text { et al. }\end{array}$ & Li et al. & RFDTI \\
\hline Enzyme & $\mathbf{0 . 9 4 7 9}$ & 0.8251 & 0.9480 & 0.9288 & 0.9172 \\
Ion channels & $\mathbf{0 . 9 3 1 2}$ & 0.8034 & 0.8890 & 0.9171 & 0.8827 \\
$\begin{array}{l}\text { GPCRs } \\
\text { Nuclear }\end{array}$ & $\mathbf{0 . 8 8 7 9}$ & 0.8235 & 0.8720 & 0.8856 & 0.8557 \\
receptors & $\mathbf{0 . 8 0 1 1}$ & 0.8394 & 0.8690 & 0.9300 & 0.7531 \\
\hline
\end{tabular}

in Table 7. It can be seen from Table 7 that the results we obtained were improved than those previously proposed methods; the increases of average AUC values on ion channel and GPCRs datasets were 0.0141, and 0.0023, respectively. However, average AUC value of the enzyme and nuclear receptor datasets are little lower than previous works, which mainly caused by the scale of prediction samples and extraction method. Generally, the comparison results demonstrated that GIST feature extraction combined with the rotation forest classifier could improve the prediction performance of drug-target interactions effectively.

\section{Conclusions}

In this article, we reported a novel computational approach combines GIST feature, position-specific scoring matrix (PSSM), and rotation forest (RF) based classification to infer unknown DTIs on a large-scale. For further evaluating the prediction ability of the proposed method, we adopted the five-fold cross-validation method on golden standard datasets. When performing on enzyme, ion channel, GPCRs, nuclear receptors, the proposed method yielded the average accuracy of $89.20 \%, 85.93 \%, 82.36 \%$, and $73.89 \%$, respectively. In order to further assess the performance of the proposed model, we made a comparison between the proposed method and the state-of-the-art support vector machine classifier. We also compare with the previous models which were 
based on the same golden standard datasets. These extensive experimental results further illustrate that the proposed method is effective and robust in predicting drug-target interactions. We expect this proposed method to be a useful tool when predicting DTIs. In future work, we plan to use more advanced feature extraction method to improve the prediction ability of DTIs.

\section{Data Availability}

The data code can be obtained at https://github.com/ TensorflowZhan/Program-Availability

\section{Disclosure}

The funders had no role in the design of the study; in the collection, analyses, or interpretation of data; in the writing of the manuscript, or in the decision to publish the results.

\section{Conflicts of Interest}

The authors declare no conflict of interest.

\section{Acknowledgments}

We are grateful to all group members in the research group led by Z.-H You for their valuable suggestions. This research is funded by the National Natural Science Foundation of China, grant numbers 61722212 and 61873212.

\section{References}

[1] P.-W. Hu, K. C. C. Chan, and Z.-H. You, "Large-scale prediction of drug-target interactions from deep representations," in International Joint Conference on Neural Networks (IJCNN), 2016.

[2] B. Niu, Y. Jin, W. C. Lu, and G. Z. Li, "Predicting toxic action mechanisms of phenols using AdaBoost learner," Chemometrics and Intelligent Laboratory Systems, vol. 96, no. 1, pp. 43-48, 2009.

[3] J. P. Overington, B. Al-Lazikani, and A. L. Hopkins, "How many drug targets are there?," Nature Reviews Drug Discovery, vol. 5, no. 12, pp. 993-996, 2006.

[4] Y. Landry and J. P. Gies, "Drugs and their molecular targets: an updated overview," Fundamental and Clinical Pharmacology, vol. 22, no. 1, pp. 1-18, 2008.

[5] B. Niu, Y.-H. Jin, K.-Y. Feng, W.-C. Lu, Y.-D. Cai, and G.-Z. Li, "Using AdaBoost for the prediction of subcellular location of prokaryotic and eukaryotic proteins," Molecular Diversity, vol. 12 , no. 1, pp. 41-45, 2008.

[6] M. Hao, S. H. Bryant, and Y. Wang, "A new chemoinformatics approach with improved strategies for effective predictions of potential drugs," Journal of Cheminformatics, vol. 10, no. 1, p. 50, 2018.

[7] X. Chen, Z. L. Ji, and Y. Z. Chen, "TTD: therapeutic target database," Nucleic Acids Research, vol. 30, no. 1, pp. 412-415, 2002.

[8] H. Yang, C. Qin, Y. H. Li et al., "Therapeutic target database update 2016: enriched resource for bench to clinical drug target and targeted pathway information," Nucleic Acids Research, vol. 44, no. D1, pp. D1069-D1074, 2016.
[9] M. Kanehisa, S. Goto, M. Hattori et al., "From genomics to chemical genomics: new developments in KEGG," Nucleic acids research, vol. 34, no. 90001, suppl_1, pp. D354-D357, 2006.

[10] S. Günther, M. Kuhn, M. Dunkel et al., "SuperTarget and Matador: resources for exploring drug-target relationships," Nucleic acids research, vol. 36, no. Database, suppl_1, pp. D919-D922, 2007.

[11] D. S. Wishart, C. Knox, A. C. Guo et al., "DrugBank: a comprehensive resource for in silico drug discovery and exploration," Nucleic acids research, vol. 34, no. 90001, pp. D668-D672, 2006.

[12] V. Law, C. Knox, Y. Djoumbou et al., "DrugBank 4.0: shedding new light on drug metabolism," Nucleic Acids Research, vol. 42, no. D1, pp. D1091-D1097, 2013.

[13] M. J. Keiser, B. L. Roth, B. N. Armbruster, P. Ernsberger, J. J. Irwin, and B. K. Shoichet, "Relating protein pharmacology by ligand chemistry," Nature Biotechnology, vol. 25, no. 2, pp. 197-206, 2007.

[14] D. L. Ma, D. S. H. Chan, and C. H. Leung, "Drug repositioning by structure-based virtual screening," Chemical Society Reviews, vol. 42, no. 5, pp. 2130-2141, 2013.

[15] D. Rognan, "Structure-Based approaches to target fishing and ligand profiling," Molecular Informatics, vol. 29, no. 3, pp. 176-187, 2010.

[16] B. Niu, Y. D. Cai, W. C. Lu, G. Z. Li, and K. C. Chou, "Predicting protein structural class with AdaBoost learner," Protein and Peptide Letters, vol. 13, no. 5, pp. 489-492, 2006.

[17] Y. Z. Chen and D. G. Zhi, "Ligand-protein inverse docking and its potential use in the computer search of protein targets of a small molecule," Proteins: Structure, Function, and Genetics, vol. 43, no. 2, pp. 217-226, 2001.

[18] Y. Tang, W. Zhu, K. Chen, and H. Jiang, "New technologies in computer-aided drug design: toward target identification and new chemical entity discovery," Drug Discovery Today: Technologies, vol. 3, no. 3, pp. 307-313, 2006.

[19] B. Niu, Y. Jin, L. Lu et al., "Prediction of interaction between small molecule and enzyme using AdaBoost," Molecular Diversity, vol. 13, no. 3, pp. 313-320, 2009.

[20] Y. Yamanishi, M. Araki, A. Gutteridge, W. Honda, and M. Kanehisa, "Prediction of drug-target interaction networks from the integration of chemical and genomic spaces," Bioinformatics, vol. 24, no. 13, pp. i232-i240, 2008.

[21] Z. Li, P. Han, Z.-H. You et al., "In silico prediction of drugtarget interaction networks based on drug chemical structure and protein sequences," Scientific Reports, vol. 7, no. 1, p. 11174, 2017.

[22] Y. Liu, M. Wu, C. Miao, P. Zhao, and X.-L. Li, "Neighborhood regularized logistic matrix factorization for drug-target interaction prediction," PLOS Computational Biology, vol. 12, no. 2, p. e1004760, 2016.

[23] N. Nagamine and Y. Sakakibara, "Statistical prediction of protein-chemical interactions based on chemical structure and mass spectrometry data," Bioinformatics, vol. 23, no. 15, pp. 2004-2012, 2007.

[24] F.-R. Meng, Z.-H. You, X. Chen, Y. Zhou, and J.-Y. An, "Prediction of drug-target interaction networks from the integration of protein sequences and drug chemical structures," Molecules, vol. 22, no. 7, p. 1119, 2017.

[25] H. Yu, J. Chen, X. Xu et al., "A systematic prediction of multiple drug-target interactions from chemical, genomic, and 
pharmacological data," PLoS ONE, vol. 7, no. 5, p. e37608, 2012.

[26] Y. A. Huang, Z. H. You, and X. Chen, "A systematic prediction of drug-target interactions using molecular fingerprints and protein sequences," Current Protein Peptide Science, vol. 19, no. 5, pp. 468-478, 2018.

[27] S. M. H. Mahmud, W. Chen, H. Jahan, Y. Liu, N. I. Sujan, and S. Ahmed, "IDTi-CSsmoteB: identification of drug-target interaction based on drug chemical structure and protein sequence using XGBoost with over-sampling technique SMOTE," IEEE Access, vol. 7, pp. 48699-48714, 2019.

[28] S. M. H. Mahmud, W. Chen, H. Meng, H. Jahan, Y. Liu, and S. M. M. Hasan, "Prediction of drug-target interaction based on protein features using undersampling and feature selection techniques with boosting," Analytical Biochemistry, vol. 589, no. 15, p. 113507, 2020.

[29] A. Ezzat, M. Wu, X. L. Li, and C. K. Kwoh, "Drug-target interaction prediction via class imbalance-aware ensemble learning," BMC Bioinformatics, vol. 17, no. S19, p. 509, 2016.

[30] I. Schomburg, "BRENDA, the enzyme database: updates and major new developments," Nucleic acids research, vol. 32, no. 90001, pp. 431D-4433, 2004.

[31] J. Shen, F. Cheng, Y. Xu, W. Li, and Y. Tang, "Estimation of ADME Properties with Substructure Pattern Recognition," Journal of Chemical Information and Modeling, vol. 50, no. 6, pp. 1034-1041, 2010.

[32] S. S.-T. Yau, C. Yu, and R. He, "A Protein Map and Its Application," DNA and Cell Biology, vol. 27, no. 5, pp. 241-250, 2008.

[33] Z. Mu, T. Yu, E. Qi, J. Liu, and G. Li, "DCGR: feature extractions from protein sequences based on CGR via remodeling multiple information," BMC Bioinformatics, vol. 20, no. 1, p. 351, 2019.

[34] X. Zhao, K. Tian, R. L. He, and S. S.-T. Yau, "Convex hull principle for classification and phylogeny of eukaryotic proteins," Genomics, vol. 111, no. 6, pp. 1777-1784, 2019.

[35] C. Yu, M. Deng, S. Y. Cheng, S. C. Yau, R. L. He, and S. S. T. Yau, "Protein space: a natural method for realizing the nature of protein universe," Journal of Theoretical Biology, vol. 318, pp. 197-204, 2013.

[36] M. Gribskov, A. D. McLachlan, and D. Eisenberg, "Profile analysis: detection of distantly related proteins," Proceedings of the National Academy of Sciences, vol. 84, no. 13, pp. 4355-4358, 1987.

[37] S. F. Altschul and E. V. Koonin, "Iterated profile searches with PSI-BLAST - a tool for discovery in protein databases," Trends in Biochemical Sciences, vol. 23, no. 11, pp. 444-447, 1998.

[38] A. Oliva and A. Torralba, "Modeling the shape of the scene: a holistic representation of the spatial envelope," International Journal of Computer Vision, vol. 42, no. 3, pp. 145-175, 2001.

[39] Y. Han and G. Liu, "A hierarchical GIST model embedding multiple biological feasibilities for scene classification," in In 2010 20th International Conference on Pattern Recognition, pp. 3109-3112, 2010.

[40] Y. Wang, Y. Li, and X. Ji, "Human Action Recognition Based on Global Gist Feature and Local Patch Coding," International Journal of Signal Processing, Image Processing and Pattern Recognition, vol. 8, no. 2, pp. 235-246, 2015.

[41] A. Torralba, K. P. Murphy, W. T. Freeman et al., "Contextbased vision system for place and object recognition," in Pro- ceedings Ninth IEEE International Conference on Computer Vision, vol. 3, 2003.

[42] H. S. Nagendraswamy, B. M. C. Kumara, and R. L. Chinmayi, "GIST Descriptors for Sign Language Recognition: An Approach Based on Symbolic Representation," in Mining Intelligence and Knowledge Exploration, pp. 103-114, 2015.

[43] C. Azzi, D. C. Asmar, A. H. Fakih, and J. S. Zelek, Filtering 3D keypoints using GIST for accurate image-based localization, BMVC, 2016.

[44] J. J. Rodriguez, L. I. Kuncheva, and C. J. Alonso, "Rotation forest: a new classifier ensemble method," IEEE Transactions on Pattern Analysis and Machine Intelligence, vol. 28, no. 10, pp. 1619-1630, 2006.

[45] B. Niu, W. C. Lu, S. S. Yang, Y. D. Cai, and G. Z. Li, "Support vector machine for SAR/QSAR of phenethyl-amines," Acta Pharmacologica Sinica, vol. 28, no. 7, pp. 1075-1086, 2007.

[46] C. C. Chang and C. J. Lin, "LIBSVM," ACM Transactions on Intelligent Systems and Technology, vol. 2, no. 3, pp. 1-27, 2011.

[47] H. Chen and Z. Zhang, "A Semi-Supervised Method for DrugTarget Interaction Prediction with Consistency in Networks," PloS one, vol. 8, no. 5, p. e62975, 2013.

[48] Z. Mousavian, S. Khakabimamaghani, K. Kavousi, and A. Masoudi-Nejad, "Drug-target interaction prediction from PSSM based evolutionary information," Journal of Pharmacological and Toxicological Methods, vol. 78, pp. 42-51, 2016.

[49] L. Wang, Z. H. You, L. P. Li, and X. Yan, "RFDTI: using rotation forest with feature weighted for drug-target interaction prediction from drug molecular structure and protein sequence," bioRxiv, 2020. 